\title{
Metabolic control through ornithine and uracil of epithelial cell invasion by Shigella flexneri
}

\author{
Correspondence \\ Glenn R. Björk \\ Glenn.Bjork@molbiol.umu.se
}

Received 11 February 2009

Revised 5 May 2009

Accepted 18 May 2009
Jérôme M. B. Durand and Glenn R. Björk

Department of Molecular Biology, Umeå University, S-90 187 Umeå, Sweden

\begin{abstract}
This paper shows that compounds in defined growth media strongly influence the expression of the effectors of virulence in the human invasive pathogen Shigella flexneri. Ornithine in conjunction with uracil reduces the haemolytic ability of wild-type cultures more than 20-fold and the expression of the type III secretion system more than 8-fold, as monitored by an mxiC: : lacZ transcriptional reporter. $m x i C$ gene expression is further decreased by the presence of methionine or branched-chain amino acids (15-fold or 25-fold at least, respectively). Lysine and a few other aminated metabolites (cadaverine, homoserine and diaminopimelate) counteract the ornithinemediated inhibition of haemolytic activity and of the expression of a transcriptional activator vir $F$ reporter. The complete abolition of invasion of HeLa cells by wild-type bacteria by ornithine, uracil, methionine or branched-chain amino acids establishes that these metabolites are powerful effectors of virulence. These findings provide a direct connection between metabolism and virulence in S. flexneri. The inhibitory potential exhibited by the nutritional environment is stronger than temperature, the classical environmental effector of virulence. The implications and practical application of this finding in prophylaxis and treatment of shigellosis are discussed.
\end{abstract}

\section{INTRODUCTION}

Many pathogens, including Shigella flexneri, express virulence factors conditionally and under tight control. This is true of invasins and the type III secretion system (T3SS) by which they are delivered into host cells. Together with various environmental conditions, nutrients are often included among factors that influence the pathogenicity of bacteria (Miller et al., 1989). The supply of the amino acid tryptophan is for example critical for intracellular pathogens such as chlamydiae (Beatty et al., 1994; Byrne et al., 1986). Four amino acids (Asp, Glu, Arg, Ser) increase the production of cholera toxin and of the pilus colonization factor TcpA in Vibrio cholerae when they are added to minimal growth medium (Miller \& Mekalanos, 1988). However, examples of molecules affecting virulent phenotypes through direct addition to the medium are scarce in both bacteria (Karlsson et al., 1999; Prunier et al., 2007; Rietsch \& Mekalanos, 2006) and pathogenic eukaryotes (Domergue et al., 2005; Fox \& Bzik, 2002; Pfefferkorn, 1984). Understandably, controlled investigations focused on deciphering the influences of gene products on other genes are designed to exclude other possible effectors, especially changes of the environment. Consequently, influences on the virulence of nutrients or other subtle and complex factors within the milieu of an in vivo infection are often neglected. The expression of virulence genes in S. flexneri,

Abbreviations: BCAA, branched-chain amino acids; DAP, meso-diaminopimelate; T3SS, type III secretion system. the causative agent of bacillary dysentery, is illustrative of a substantial role of the environment on pathogenesis. Its requirements for the modification Q34 in the tRNA differ according to specific substances present in or absent from the growth medium (Durand \& Björk, 2003). The stimulatory role on virulence gene expression conveyed by putrescine, or by a mixture of the metabolically related arginine and methionine, in the growth medium suggests nutrient-specific influence of pathways for the invasive behaviour of $S$. flexneri. This effect provides a route to better understanding fundamental yet underexplored aspects of metabolites on pathogenicity.

The facultative intracellular bacterium $S$. flexneri, which is very closely related to Escherichia coli, destroys the mucosa of the colon in humans and monkeys by invading and replicating in epithelial cells and by moving between such cells. Extensive destruction leads to a characteristic bloody diarrhoea which may favour the dissemination of the pathogen. Invasion is promoted by the activation of regulatory elements borne on a $230 \mathrm{kbp}$ large virulence plasmid and on the bacterial chromosome. The determinants for the effectors of the virulence are also borne on the plasmid. They include the determinants for the invasins $\mathrm{IpaB}, \mathrm{C}, \mathrm{D}$ and the elements of a T3SS involved in the correct delivery of the virulence effectors into the host cell and secreted into the surrounding milieu. Through direct interactions with cellular targets, these effectors influence various cellular signalling pathways and perturb the innate immune activation of the host (Ogawa \& Sasakawa, 2006). 
Virulence genes are functionally organized in a cascade, which, under the right environmental conditions, amplifies the expression of invasins 100-200-fold. The virF gene, the most upstream gene in this cascade, encodes an AraCfamily transcriptional activator. It is required for the expression of the virulence effector VirG, which is responsible for the intracellular motility of Shigella, manifested through its ability to polymerize actin molecules of the cytoskeleton of the host cells. The VirF protein also activates the expression of VirB, another transcriptional activator and member of the ParB family, involved in plasmid partitioning. The $\operatorname{VirB}$ protein is required for induced expression of the ipa operon and for the activation of the genes encoding the proteins constituting the T3SS apparatus. The influences of temperature, osmolarity and $\mathrm{pH}$ on the expression of the virulence genes have been extensively studied (Maurelli et al., 1984; Nakayama \& Watanabe, 1995; Porter \& Dorman, 1994), and this pathogen is consequently considered a paradigm in virulence gene regulation.

Acquired through oral contamination, S. flexneri crosses the different parts of the digestive tract before reaching the terminal part of the colon where disease typically ensues. Since expression of the virulence genes is a prerequisite for cellular invasion, the growth conditions must trigger the expression of these genes. Indeed, the expression of the effectors of virulence decreases as growth conditions deviate from those prevailing in the lower gut, including $37{ }^{\circ} \mathrm{C}$, pH near 7.4, or osmolarity of saline. Temperature, osmolarity and $\mathrm{pH}$ exert a cumulative influence on the transcription of the virF gene (Dorman \& Porter, 1998). Variations in the concentration of VirF correlate with variations in the expression of invasins and occur in parallel with changes in environmental conditions that affect transcription of the virF gene. Mutants defective in the formation of VirF do not express virulence-associated genes in culture. Thus, there is a close correlation between the synthesis of VirF and expression of virulence-associated phenotypes, such as the invasion of HeLa cells and the contact haemolytic assay.

Interestingly, the efficiency with which the virF mRNA is translated into the VirF polypeptide is also critical for the expression of virulence genes. The integrity of the tRNA modification genes tgt and miaA, responsible for the synthesis of the modified nucleosides queuosine (Q) and 2-methylthio- $N^{6}$-isopentenyladenosine $\left(\mathrm{ms}^{2} \mathrm{i}^{6} \mathrm{~A}\right)$ respectively, is a prerequisite for full expression of VirF and thereby of the invasins. The translation of the virF mRNA can modulate the environmental signal very tightly. Thus, although virF transcription is similar to that found in the wild-type, miaA::KmSma mutants of $S$. flexneri exhibit a total inhibition of virulence gene expression at $37{ }^{\circ} \mathrm{C}$ grown in rich undefined medium (Durand et al., 1997), and a tgt mutant shows the same effect grown in glucose minimal medium (Durand \& Björk, 2003). This post-transcriptional step should be considered in the analysis of the stimulation provided by the environment, not only quantitatively but also qualitatively, since a regulation at this step also allows the input of additional regulatory influences. There is as yet no clear experimental evidence for the mediation of an environmental influence on the expression of virulence genes through variations in the level of the tRNA modification. However, the production of VirF in the tgt mutant and subsequently the production of invasins is clearly dependent on the presence of amino acid mixtures in the growth medium, and the specific requirement of arginine plus methionine has been demonstrated (Durand \& Björk, 2003). These enrichments, however, do not influence virulence gene expression in the wild-type. The medium-dependent expression of virulence genes observed in the tgt mutant suggests that difference in the degree of tRNA modification and presence of specific nutrients could shape the virulence response mounted by S. flexneri. Indeed, genetic analyses dedicated to the identification of mutants with decreased invasivity have shown that biosynthetic pathways such as those for methionine biosynthesis (Rothman \& Corwin, 1972; Zagaglia et al., 1991) or guanine biosynthesis (Cersini et al., 1998; Noriega et al., 1996) are involved in the process of invasion. Although these studies did not identify the metabolic intermediates influencing invasion, quinolinate, which is an intermediate in NAD biosynthesis, was recently reported to inhibit invasion (Prunier et al., 2007). Nevertheless, a gap exists in the wider understanding of how metabolic pathways may influence virulence gene expression in S. flexneri. Screening for molecules active on the haemolytic ability of wild-type $S$. flexneri, we have found that ornithine (Orn) and uracil (Ura) in the medium severely reduce virulence gene expression and, surprisingly, to the same degree as does reduced temperature. Further supplementations identified specific metabolites in the growth medium that can act as regulators of the pathogenicity of Shigella as strong as the more established environmental conditions of temperature, osmolarity and $\mathrm{pH}$.

\section{METHODS}

Bacterial strains and plasmids. Wild-type S. flexneri 2a YSH6000 (Sasakawa et al., 1986) was used together with the virulent strain 2457T (Formal et al., 1971). Strain N1436, the congenic tgt::Tn5 derivative of YSH6000, has been described (Durand et al., 1994; Okada et al., 1991). Plasmid pHW848 (virF' -'lacZ) was provided by H. Watanabe (Nakayama \& Watanabe, 1995). Strain BS184, a 2457T derivative harbouring the mxiC:: lac $Z$ fusion, was provided by $\mathrm{A}$. Maurelli (Maurelli \& Sansonetti, 1988).

Growth media. Bacteria were grown at $37^{\circ} \mathrm{C}$ in the rich medium LB (Miller, 1972). Glucose minimal medium buffered with MOPS (Neidhardt et al., 1974) was supplemented with nicotinic acid $(2.5 \mu \mathrm{g}$ $\mathrm{ml}^{-1}$ ). In enrichment experiments individual components of the defined rich MOPS medium (Neidhardt et al., 1977) were added to minimal MOPS glucose medium. L-Ornithine monohydrochloride (Sigma), cadaverine, putrescine and homoserine were added to the growth media at a concentration of $10 \mathrm{mM}$.

Virulence phenotype assay. In the contact haemolytic assay the absorbance at $545 \mathrm{~nm}$ of the supernatant of an incubation mix of 
bacteria with sheep erythrocytes reflects the production of invasins (Sansonetti et al., 1986). Haemolytic activity is expressed as a percentage relative to the haemolytic activity expressed by the wildtype grown to late-exponential phase in a reference medium, either unsupplemented minimal MOPS or LB. Variations in the absolute level of haemolysis can occur from experiment to experiment. Therefore, in each experiment, the haemolytic activity was always related to the activity in the wild-type. Moreover, comparisons between the relative levels of haemolysis in different experiments (each being done in at least four replicates) should take this point into account. Additions of the various nutrients did not grossly affect the growth of the bacteria.

For the invasion assay, bacteria were grown as indicated in the 'Growth media' section above before exposing them to HeLa cells according to conditions described previously (Hale \& Formal, 1981); $1.2 \times 10^{6} \mathrm{HeLa}$ cells were inoculated with bacteria, and infection was initiated by centrifuging the plates at $700 \mathrm{~g}$ for $10 \mathrm{~min}$. After incubating the cells at $37{ }^{\circ} \mathrm{C}$ for $30 \mathrm{~min}$ in the presence of $5 \% \mathrm{CO}_{2}$, the culture medium was replaced with a medium supplemented with $200 \mu \mathrm{g}$ gentamicin $\mathrm{ml}^{-1}$, and incubated for an additional $120 \mathrm{~min}$. After washing the cells with ice-cold PBS twice and lysing them in PBS supplemented with $0.5 \%$ Triton X-100, the internalized bacteria were counted by plating serial dilutions of the lysates on agar plates, which were incubated at $37{ }^{\circ} \mathrm{C}$ overnight.

Immunoblotting. Bacteria grown in the various media were sampled at the end of the exponential phase of growth and their quantity was normalized according to the optical density of the culture. The cells were washed, and then boiled for 3 min in sample buffer prior to SDS-PAGE. Immunoblotting of SDS-PAGE-resolved antigens was performed as described by Nakata et al. (1992). The VirF-specific antiserum HALLON has been described previously (Durand et al., 2000). The antibodies directed toward Ipa proteins have been described (Pál et al., 1989). Detection of immune complexes was performed with either the ECL or the ECF system (Amersham Life Science); bands of interest were quantified via the appropriate scanning methodology.

Analysis of content of modified nucleosides in tRNA. Strains were grown in the appropriate medium at $37{ }^{\circ} \mathrm{C}$ and harvested at a density of approximately $4 \times 10^{8}$ cells $\mathrm{ml}^{-1}$. tRNA was prepared by phenol extraction (Emilsson \& Kurland, 1990), purified on a Nucleobond column, and digested to nucleosides by nuclease P1 and alkaline phosphatase (Gehrke et al., 1982). The hydrolysate was analysed by HPLC (Gehrke \& Kuo, 1989).

\section{RESULTS}

\section{Ornithine in the growth medium reduces the haemolytic activity of wild-type $S$. flexneri}

The stimulatory influence of arginine (Arg) plus methionine (Met) and of the polyamine putrescine on the virulence system of $S$. flexneri has been evidenced previously (Durand \& Björk, 2003). Although this nutritional stimulatory effect was observed in a mutant background (tgt) lacking queuosine $(\mathrm{Q})$ in the tRNA, this observation prompted us to test the response mediated by metabolites of arginine-methionine-polyamine metabolism on the virulence gene expression of wild-type $S$. flexneri. Ornithine (Orn) is a precursor in the biosynthetic pathway leading to Arg and is also the starting metabolite in the synthesis of the polyamine putrescine, which is converted to another polyamine, spermidine, by transfer of a methyl group from the Met derivative $S$-adenosylmethionine (Fig. 1). In contrast to the stimulation of virulence gene expression in the tgt mutant mediated by the Arg + Met mixture or by putrescine (Durand \& Björk, 2003) (see also Fig. 2a, bars 1, 2, 3, 4), the addition of Orn to the minimal growth medium triggered a decreased haemolytic ability of the wild-type S. flexneri (Fig. 2a, bar 5). Testing different concentrations of Orn (0.1-20 mM), a dose response was observed between 1 and $10 \mathrm{mM}$ Orn (data not shown; no response was observed using $0.1 \mathrm{mM}$, and $20 \mathrm{mM}$ did not increase the inhibition compared to $10 \mathrm{mM}$ ). We conclude that although the stimulatory effects of Arg + Met and putrescine are not detected in the wild-type by monitoring the haemolytic ability, the expression of virulence genes is sensitive to the composition of the growth medium also in wild-type $S$. flexneri.

The influence of the growth medium on virulence required further exploration of this Orn-mediated nutritional inhibition of haemolysis. Supplementation of the Orncontaining medium with the active compounds identified previously was therefore undertaken. Whereas putrescine completely counteracted the Orn-mediated inhibition, the Arg + Met mixture did not (Fig. 2a, bar 9 black and bar 6). Met alone reinforced the inhibition mediated by Orn (Fig. 2a, bar 8), whereas Arg alone showed, on the contrary, a slight counteracting effect on the inhibitory effect of Orn (Fig. 2a, bar 7, black). However, this Arg-mediated effect was far less than the counteracting effect provided by the presence of Arg + Met mixture in mutant cultures (Fig. 2a, white bars 2 and 4). The difference in the haemolytic activity expressed in the two Orn-containing cultures (Orn + Met versus Orn + putrescine; compare Fig. 2a, black bars 8 and 9) was more than sixfold. Clearly, the nutritional context strongly influences the haemolytic ability of the wild-type, suggesting that virulence gene expression is triggered by the presence of specific nutrients, which can be either stimulatory or inhibitory.

\section{Orn in the growth medium does not influence tRNA modification}

Since lack of $\mathrm{Q}$ in tRNA, as in the tgt mutant, reduces virulence gene expression, the observed Orn effect might be caused by a reduced level of Q in the tRNA of the wildtype. However, this is not the case, since modification of tRNA in wild-type $S$. flexneri was independent of Orn in the growth medium (data not shown). Thus, the decreased virulence observed in the presence of Orn in the wild-type cells was not mediated by undermodification of the tRNA.

\section{Uracil and branched-chain amino acids in the growth medium increase the inhibitory effect of Orn on haemolysis}

Orn is a precursor for putrescine (Fig. 1). Putrescine, as shown above, counteracts the Orn-mediated inhibition. 


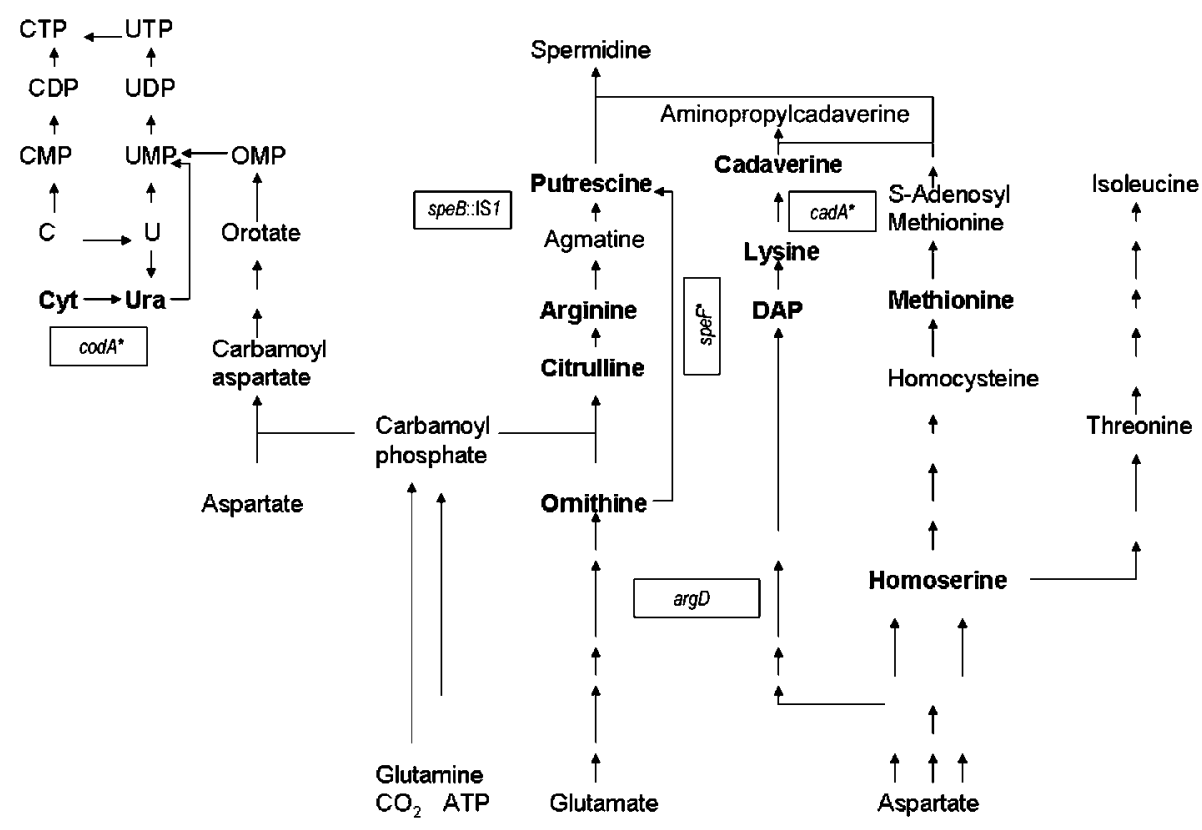

Fig. 1. Metabolic relationships of nutrients (in bold) monitored in this study for their role in the expression of virulence genes of S. flexneri. This scheme is drawn from the knowledge in E. coli (Neidhardt et al., 1996). The nutritional compounds identified in this study for their activity on virulence-associated phenotypes indicate the involvement of the arginine and polyamine biosynthetic pathways as well as intermediates in the pyrimidine, lysine and methionine pathways. In these pathways, the genes $\operatorname{cod} A$, spe $F$ and $\operatorname{cad} A$ are present in $E$. coli but absent in $S$. flexneri. As has been suggested for cad $A$, the absence of these genes, which are marked with *, could be related to the pathoadaptative evolution which led from commensal $E$. coli to modern Shigella (Casalino et al., 2003; Maurelli et al., 1998), supporting the critical role of uracil, putrescine and cadaverine in virulence gene expression identified in this study. In addition, $\arg F$, which encodes one of the isozymes for ornithine carbamoyltransferase, is absent in $S$. flexneri and a transposable element IS1 alters the promoter region of the gene speB, responsible for the conversion of agmatine to putrescine. Since astC, encoding the acetylornithine aminotransferase isozyme in $E$. coli, is also absent in $S$. flexneri, the gene $\arg D$, encoding a protein with two activities, acetylornithine aminotransferase and $N$ succinyldiaminopimelate aminotransferase, could coordinate the biosynthesis of arginine/ornithine and of lysine in $S$. flexneri.

Orn is also the precursor for Arg and citrulline. As shown above, Arg shows only a minor counteracting effect on the inhibition of virulence gene expression exerted by Orn, and citrulline supplementation does not show a significant difference (Fig. 2b, bar 3). However, in the biosynthetic pathway leading to arginine, the transformation of Orn into citrulline involves carbamoyl phosphate (Glansdorff, 1996). This compound otherwise feeds the conversion of aspartate to carbamoyl aspartate at the first step of the pathway leading to pyrimidine synthesis (Fig. 1). Accordingly, the pyrimidine and arginine biosynthetic pathways share mutual regulatory influences, providing our rationale for examining the possible involvement of pyrimidines in the expression of virulence genes.

We added the bases uracil (Ura) or cytosine (Cyt) together with Orn to the growth medium (Fig. 2b, bars $4,5,6$ ). To our surprise, Ura further reduced Orn-mediated inhibition of the haemolytic activity from $20-33 \%$ to only $5 \%$ of the level expressed in the wild-type (Fig. 2b, bar 4). As by adding Orn alone to the growth medium, a dose response was observed also in the presence of $0.2 \mathrm{mM}$ Ura (data not shown). On the other hand, Cyt had a slight counteracting effect on the Orn-mediated inhibition (Fig. 2b, bar 6). Addition of Ura or Cyt without Orn to minimal MOPS medium showed only a slight (Ura) or no (Cyt) decrease of the haemolytic activity of the wild-type strain YSH6000. Thus, the Orn-mediated inhibition of virulence gene expression is reinforced by the presence of Ura but not by Cyt.

We looked for other nutrients able to further decrease the haemolytic activity of the cultures in conjunction with the Orn + Ura mixture. Met, which reinforced slightly the Ornmediated decrease on this virulence associated phenotype (Fig. 2a, bar 8, black), acted synergistically with the Orn + Ura mixture (Fig. 2b, bar 7). Screening of other components of the rich MOPS medium showed that branched-chain amino acids (Val+Ile + Leu: BCAA) together with the Orn + Ura mixture reduced the haemolytic ability as much as Met and to the limit of detection (Fig. 2b, bar 8). Val added alone to the Orn + Ura mixture also reduced the haemolytic activity to the level of detection (Fig. 2b, bar 10), suggesting that Val could be the active compound of the BCAA mixture. However, Val did not influence the reduction of the haemolytic activity 


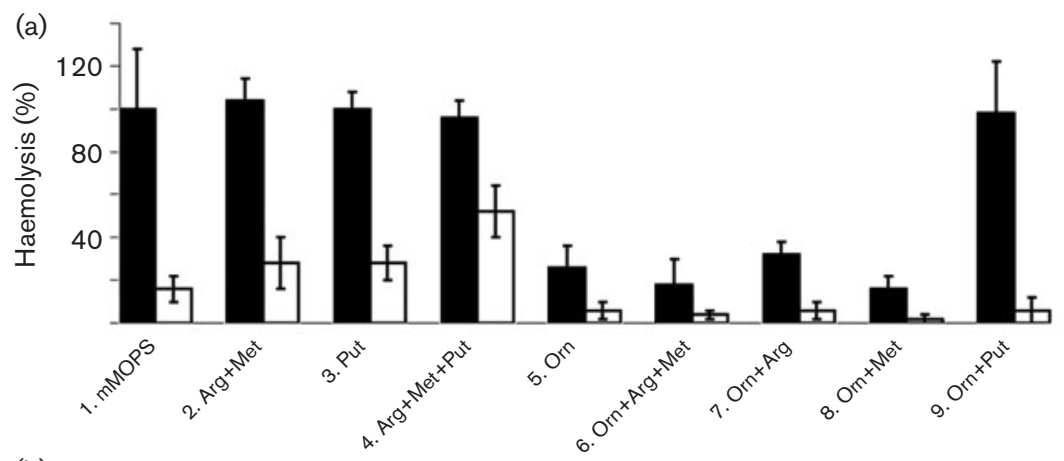

(b)

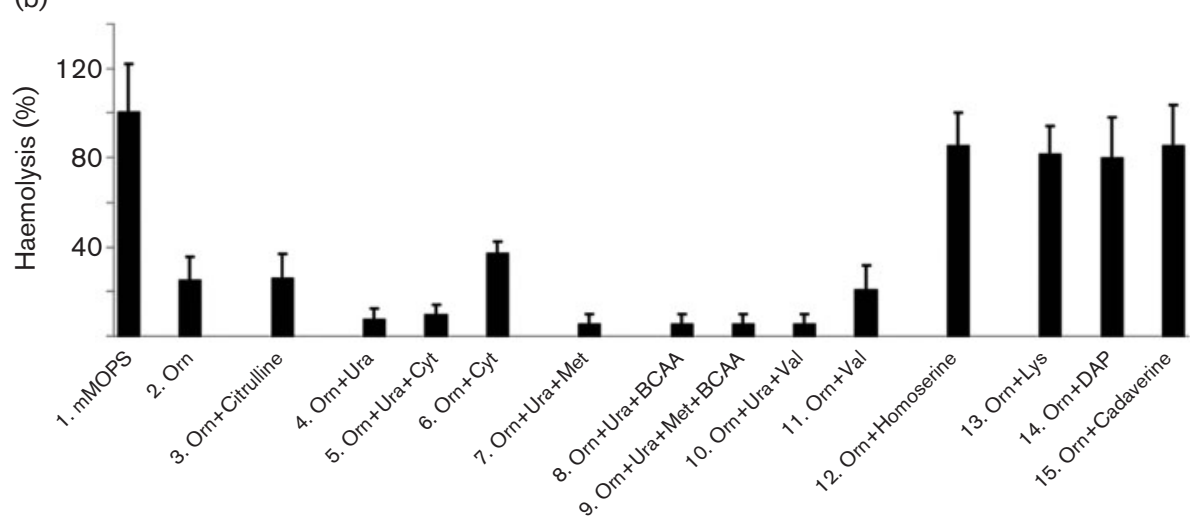

Fig. 2. The haemolytic activity of $S$. flexneri depends on the presence of a few specific nutrients in the growth medium. (a) Haemolytic activity of strain YSH6000 (wild-type, black bars) and of the tgt mutant (white bars) growing in minimal MOPS glucose medium, unsupplemented (1) or supplemented as indicated (2-9), differs several fold according to the presence of nutrients metabolically related to each other and to the biosynthesis of polyamines. (b) Haemolytic activity of wild-type strain YSH6000 growing in the presence of ornithine (bars 2-15) and supplemented with the indicated compounds. The data are presented in groups according to the metabolic relationship of the supplements: bars 4-6, pyrimidines; bars 8-11, BCAA (Val, Leu and Ile); bars 13-15, lysine. Additions of these compounds to minimal medium without Orn did not affect the haemolytic activity except for Ura and Cyt + Ura, which reduced haemolysis of the wild-type strain 2457 T to $70-100 \%$ of that observed in the unsupplemented medium (data not shown).

induced by Orn (Fig. 2b, bar 11). Since these supplementations of the Orn- and Ura-containing medium inhibited the haemolytic activity of wild-type bacteria almost completely (Fig. 2b, bars 7-10), we tested the expression of the T3SS reporter construct mxiC:: lacZ in order to further confirm the effect of Orn in conjunction with Ura, Met and BCAA. Not only did Orn reduce similarly the haemolytic ability and the transcription of the $m x i C$ gene (Fig. 3, bar 2) but also the addition of Ura, Met or BCAA in conjunction with Orn reduced further the haemolytic activity and $m x i C$ transcription to the same degree (Fig. 3, black and grey bars $3,7,11,14$, and 15). However, added to the medium without Orn, these compounds did not show any influence neither on the haemolytic ability nor on transcription (Fig. 3, black and grey bars 4, 5, 8, 9, 12, 13, and 16).

Substances counteracting the Orn-mediated inhibition of the haemolytic activity include homoserine and lysine (Lys) as well as the Lys-related compounds meso-diaminopimelate (DAP) and cadaverine.
Homoserine is an intermediate in the synthesis of Met and BCAA (Fig. 1). Since these compounds are clearly involved in the expression of the virulence genes, we explored the related metabolite homoserine for its possible involvement on the haemolytic ability in the wild-type in either the presence or absence of Orn. In contrast to the reinforcement of Orn-mediated inhibition of the haemolytic activity by Met (Fig. 2a, black bar 8), added homoserine was counteractive, and re-established haemolytic activity to the wild-type level (Fig. 2b, bar 12). Thus, homoserine, like putrescine (Fig. 2a, black bar 9), relieved the inhibition of virulence mediated by Orn.

In an undefined rich medium (LB), addition of Orn or Orn + Ura did not influence virulence gene expression of the wild-type strain (data not shown). Similarly, Orn did not influence the virulence gene expression of the wild-type strain in a defined rich medium (rich MOPS). It is possible that additional nutrients in the rich media also impinge on virulence gene expression. Defined rich MOPS medium contains all 20 amino acids, all four nucleobases and a 


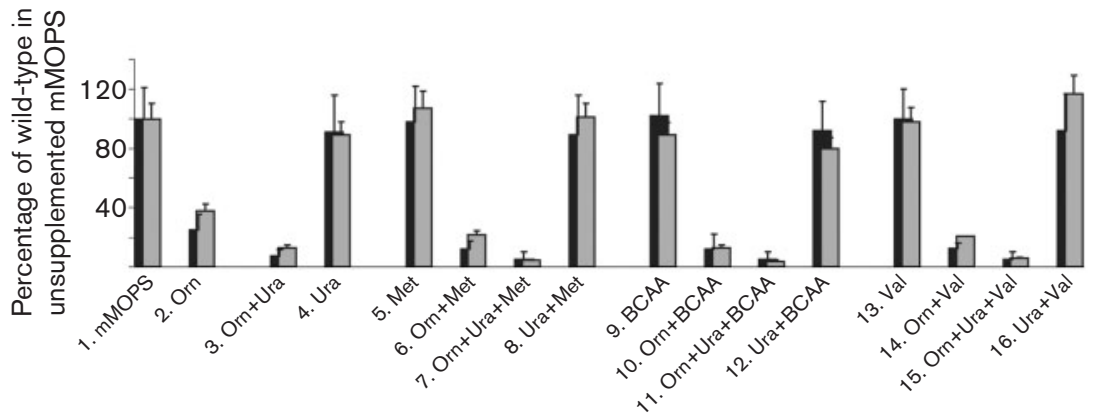

Fig. 3. The expression of the T3SS is reduced to a similar extent as the haemolytic ability by the composition of the growth medium. The expression of the T3SS was monitored by the expression of $m x i C$ : : lac $Z$ (grey bars). The data are presented as percentage of the $\beta$-galactosidase activity in unsupplemented glucose minimal MOPS medium. For comparison the haemolytic activity (Fig. 2b) is shown (black bars).

vitamin mixture (Neidhardt et al., 1977), but does not contain putrescine or homoserine. We therefore initiated a systematic search for the metabolite(s) exerting this counteracting effect present in rich MOPS medium (data not shown). It revealed Lys alone as the counteractive agent (Fig. 2b, bar 13). We further tested metabolites that are part of the lysine pathway but not present in the rich MOPS medium. meso-Diaminopimelate (DAP), the direct biosynthetic precursor of Lys, as well as cadaverine, a degradation product of Lys, also counteracted the Ornmediated inhibition of the haemolytic activity (Fig. 2b, bars 14 and 15).

In summary, the identified metabolites that were able to counteract the Orn-mediated inhibition of virulence gene expression are directly or indirectly associated with the biosynthesis of polyamines. These results illustrate the importance of the quality of the growth medium on the virulence response elicited by S. flexneri. Clearly, the influence of nutrition on virulence, as shown previously for the tgt mutant (Durand \& Björk, 2003), is not an esoteric phenotype shown by a mutant but it is an intrinsic behaviour of wild-type bacteria.

\section{Orn and Ura rival temperature as powerful effectors of virulence gene expression for $S$. flexneri}

To establish the effect of the nutritional environment on virulence gene expression at the molecular level, we monitored the expression of Ipa proteins by Western blot analysis (Fig. 4). Orn + Ura decreased the level of Ipa proteins more than did growth at $30{ }^{\circ} \mathrm{C}$. Addition of Met or of BCAA to cultures containing the Orn + Ura mixture (Fig. 4) further lowered the level of IpaC, already strongly reduced by the Orn + Ura mixture. Val added alone to the Orn + Ura mixture (Fig. 4) also reduced IpaC, suggesting here also that Val could be the active compound in the mixture of BCAA. Thus, the decrease of haemolytic activity induced by the various metabolites was correlated with a corresponding decrease of the invasins IpaB, C and D.

The decrease in the expression of invasins occurring when Orn, Ura, Met and BCAA were included in the defined medium was tested for its biological relevance by determining the ability of the bacteria to invade human cells. As expected from the level of invasins, Orn, Ura, Met and BCAA together inhibited the invasivity of the prototypic wild-type strains YSH6000 and 2457T in the gentamicin killing assay to a greater degree than that induced by low temperature $\left(30^{\circ} \mathrm{C}\right)$ (Fig. 5). Thus, a correct choice of supplements to the growth medium demonstrates that the inhibitory potential of invasion exhibited by the nutritional environment is stronger than temperature, the classical environmental effector of virulence.

\section{Orn alone at $37{ }^{\circ} \mathrm{C}$ and growth at $30^{\circ} \mathrm{C}$ reduce virF transcription to the same degree}

We have shown previously that temperature, tRNA modification and DNA superhelicity change the level of the key regulatory protein VirF present in the bacteria (Durand et al., 2000). We therefore suspected that the nutrients also acted primarily by altering the expression of VirF, targeting both the expression of invasins and the expression of the T3SS. Indeed, addition of Orn to the defined glucose minimal medium decreased by one-third the level of the VirF protein as monitored by Western blotting (Fig. 6). The Orn + Ura mixture inhibited the expression of VirF by about two-thirds, which is much

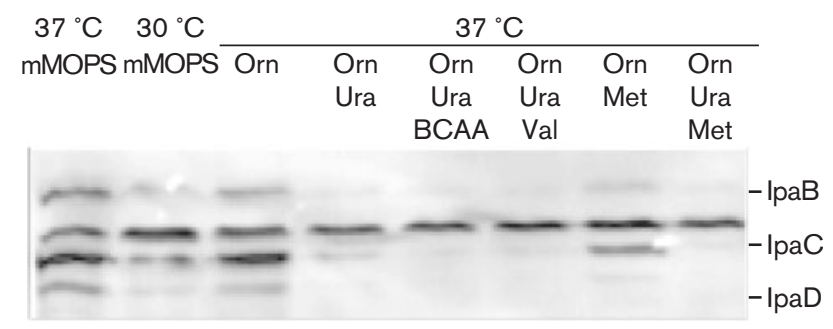

Fig. 4. The composition of the growth medium controls the levels of invasins: Western blot analysis of the expression of the effectors of the invasins IpaB, C and D in the wild-type S. flexneri strain YSH6000 growing in minimal MOPS glucose medium or in media supplemented by the indicated nutrients at $37^{\circ} \mathrm{C}$ or at $30{ }^{\circ} \mathrm{C}$. The band of intermediate molecular mass between IpaB and IpaC revealed by the immunostaining is not involved in invasion and serves as an internal control. 


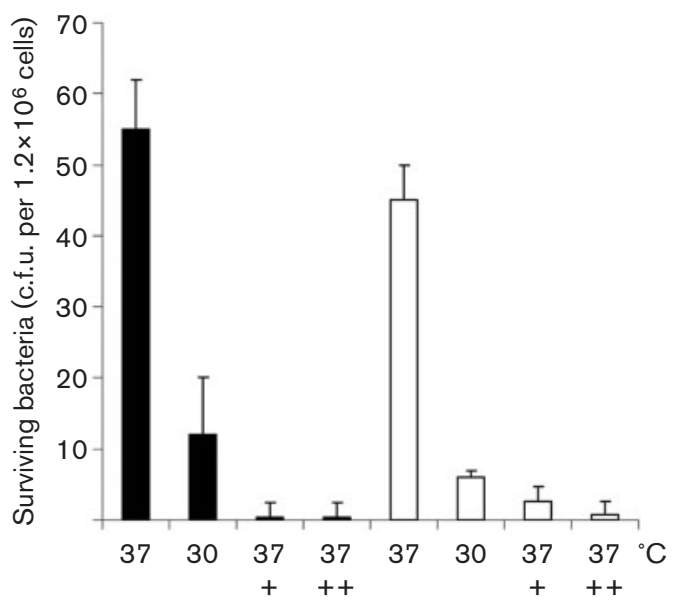

Fig. 5. The composition of the growth medium controls invasion. The nutritional environment inhibits invasion more efficiently than growth at $30{ }^{\circ} \mathrm{C}$, as expected from the lowered levels of invasins (Fig. 4). Bacteria grown at $30{ }^{\circ} \mathrm{C}$ or $37{ }^{\circ} \mathrm{C}$ in minimal MOPS glucose medium supplemented or not with inhibitory nutrient mixtures [Orn, Ura, Met, Val (+) or Orn, Ura, Met, Val, Leu, lle $(++)]$ and normalized to the same optical density were used to infect HeLa cell monolayers. Black bars, wild-type strain YSH6000; white bars, wild-type strain 2457T.

more than the effect seen with Orn alone (Fig. 6) and consistent with the lower haemolytic activity exhibited by Orn + Ura compared to Orn alone (Fig. 2b, bars 2 and 4). Addition of Met to Orn-containing minimal medium inhibited the expression of $\mathrm{VirF}$ as compared to the medium supplemented with Orn alone, but to a lesser extent than the mixture of Orn and Ura (Fig. 6). Val in combination with Orn did not reduce the level of VirF more than Orn alone, although the haemolysis was slightly reduced (Fig. 2b, bars 2 and 11), consistent with the reduction of the reporter mxiC: : lacZ (Fig. 3, bar 14 grey). We note that the level of VirF in a culture growing at $37{ }^{\circ} \mathrm{C}$ in the presence of Orn + Ura is equivalent to that in a culture growing at $30^{\circ} \mathrm{C}$ (Fig. 6). Thus, the effect exerted

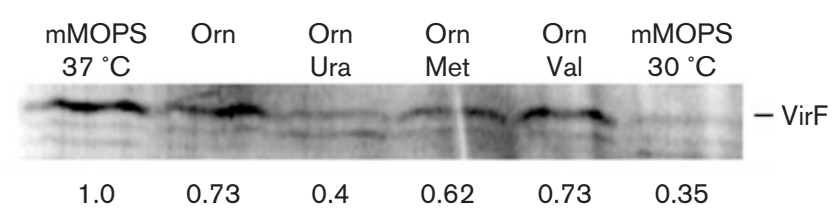

Fig. 6. The level of the VirF protein parallels the influence of the nutritional environment on virulence gene expression: Western blot analysis of the expression of $\mathrm{VirF}$ in the presence of the nutrients that influence haemolytic activity of strain YSH6000. A representative experiment is shown out of three done. Below each lane the level of VirF resulting from scanning (Image Quant) is reported relative to the level found in MOPS medium. by the various metabolites on the haemolytic activity was correlated with a reduced level of VirF, the master regulator of virulence in Shigella. However, an appreciable amount of VirF (one-third of the amount in minimal MOPS at $37{ }^{\circ} \mathrm{C}$ ) was still detected when the haemolysis was brought to the lowest level either by the temperature (Fig. 6 , mMOPS $30{ }^{\circ} \mathrm{C}$ ) or by the nutrients (Fig. 6, Orn Ura), suggesting that the metabolites could affect the cascade of events leading to the expression of invasins also independently of the VirF-mediated effect.

Osmolarity (Porter \& Dorman, 1997), pH (Nakayama \& Watanabe, 1995) and temperature (Tobe et al., 1991) influence transcription of virF. Therefore, we monitored its transcription in the presence of metabolites influencing the haemolytic activity. Using the virF'-lac $Z$ fusion (Nakayama \& Watanabe, 1995), built previously to investigate the transcriptional regulation of virF expression by the $\mathrm{pH}$, we monitored the $\beta$-galactosidase activity expressed by this reporter in minimal MOPS medium in the presence or absence of Orn (Fig. 7). Orn caused a 50 $70 \%$ decrease of the $\beta$-galactosidase activity, i.e. to a similar degree as the reduction of the haemolytic activity and as the level of VirF protein as monitored by Western blotting. We concluded that Orn in the growth medium acted, like $\mathrm{pH}$ and temperature, on the transcription of virF.

The addition of Ura, Met or BCAA to the Orn-containing medium has synergistic effects on the inhibition of haemolysis (Fig. 2b, bars 2, 4, 7, 8), of Ipa production (Fig. 4), and of expression of the type III reporter mxiC:: lacZ (Fig. 3, grey bars), but the extent of reduction of the expression of the $\operatorname{vir} F^{\prime}-{ }^{\prime}$ lac $Z$ reporter was less than that for genes believed to be under the direct control of VirF (Fig. 7). Thus, Ura, Met and BCAA may influence the expression of VirF post-transcriptionally or act directly on the genes downstream of VirF in the regulatory cascade of virulence in Shigella.

As expected, homoserine, Lys, DAP and cadaverine, which counteracted the Orn-mediated decrease in haemolysis (Fig. 2b, bars 12-15), also counteracted the Orn-mediated reduction of virF transcription (Fig. 7).

\section{DISCUSSION}

Here we report a novel manifestation of how the environment influences the expression of virulence genes of the human pathogen S. flexneri. A few specific metabolites unexpectedly show a strong regulatory effect on the invasivity of wild-type S. flexneri YSH6000 as well as on the other prototypic wild-type strain 2457T (data not shown). Orn reduced 3-10-fold the haemolytic ability of wild-type strains (see Fig. 2b, bar 2 for strain YSH6000), and decreased accordingly the expression of the $v_{i r} F^{\prime}-{ }^{\prime} l a c Z$ gene fusion and the level of VirF, which is the upstream regulator of virulence in S. flexneri. Addition of Ura or Met reinforced the Orn-mediated reduction of the haemolytic activity five- and twofold, respectively (Fig. 2b, bars 2, 4; 


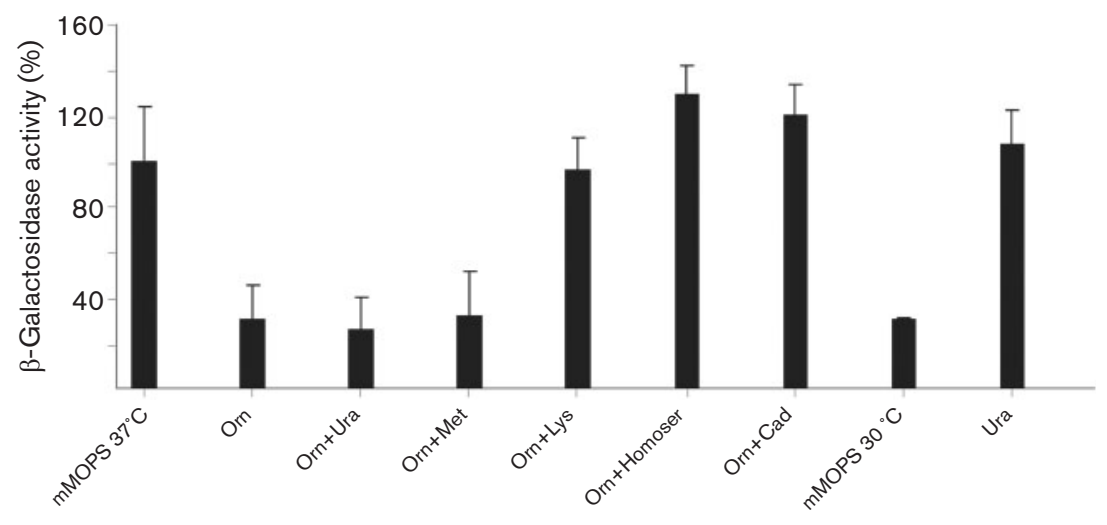

Fig. 7. The transcription of the virF gene is inhibited by the presence of ornithine in the growth medium, and this inhibition is modulated in the presence of nutrients which interfere with the ornithine inhibition of the expression of virulence genes. The $\beta$-galactosidase activity of the wild-type strain 2457T transformed with plasmid $\mathrm{pHWH848}$ virF' $^{-}$ ' $/$ acZ and growing in minimal MOPS glucose medium supplemented with the indicated nutrients or temperature is reported as a percentage of the value in minimal MOPS glucose medium at $37^{\circ} \mathrm{C}$.

Fig. 2a, black bars 5, 8) and decreased further the expression of $m x i C$ : : lac $Z$ three- and twofold, respectively (Fig. 3). Apparently these reinforcing inhibitory metabolites are operating downstream of virF transcription, since they did not reduce further the transcription of the virF gene beyond the reduction caused by Orn alone (Fig. 7). Orn, Ura and Met together with Val, which has no notable activity by itself (Fig. 2b, bar 11), inhibited the haemolytic activity of the bacteria to a non-detectable level (Fig. 2b, bars 7-10). Consistently, in defined medium they abolished the expression of invasins more efficiently than growth at $30{ }^{\circ} \mathrm{C}$ (Fig. 4) as well as the expression of the T3SS (Fig. 3). Consequently, the ability of the bacteria to invade cultured cells is completely impaired by the presence of just these few metabolites (Fig. 5). These influences are mediated by a reduction of the intracellular level of VirF (Fig. 6), which correlates with changes observed at the level of the haemolytic activity. Orn alone decreases the expression of the vir $F^{\prime}-$ lac $Z$ gene fusion to the same degree as other well-documented physiological conditions known to act transcriptionally, such as temperature and $\mathrm{pH}$.

Except Orn, the metabolites assayed in this study added alone to the growth medium did not affect the virulence of wild-type $S$. flexneri (data not shown). The active metabolites either reinforced the Orn-mediated inhibition of virulence gene expression (e.g. Ura, Met) or counteracted it (e.g. Lys, homoserine, cadaverine; see below). Thus, Orn was the only metabolite that alone substantially reduced the haemolytic activity (Fig. $2 \mathrm{a}$ and legend to $2 \mathrm{~b}$ ), the type III reporter fusion (Fig. 3), the expression of the $\operatorname{vir} F^{\prime}-{ }^{\prime}$ lacZ gene fusion (Fig. 7), and the level of the VirF protein (Fig. 6). These facts identify Orn as a unique metabolite in the regulation of expression of virulence genes. The amino acid Orn is not present in proteins, but is an intermediate in the biosynthesis of arginine and of putrescine in E. coli (Fig. 1) and also in their degradative pathways.

The counteracting effect of Lys on Orn-mediated inhibition may explain the inefficiency of Orn in LB, since LB contains many uncharacterized substances. To be taken up by the bacterium, Lys and Orn share a common transporter which has a stronger affinity for Lys than for Orn (Celis et al., 1973). Therefore, Orn could be excluded from entering the cell by Lys, explaining the counteracting effect of Lys on Orn inhibition of virulence gene expression. However, DAP also belongs to the lysine pathway but uses a different transporter (Berger \& Heppel, 1972). DAP counteracts the Orn effect by re-establishing the level of haemolysis to that exhibited in minimal medium in the wild-type (Fig. 2b, bar 14). Therefore, although the Lys effect may be caused by inhibition of Orn uptake, the DAP effect suggests that a direct influence on the metabolism by Lys is causing the observed effect. It is likely that metabolic pathways leading to and from DAP are inter-regulated in S. flexneri, since this cell wall component plays a critical role during host cell invasion and is later essential for intracellular survival (Xu \& Ulmer, 2003). Our discovery of convergent and opposing influences by the Lys and Orn biosynthetic pathways reveals direct control by nutrients in S. flexneri. These findings provide solid evidence and a rationale for a functional direct connection between metabolism and virulence in this bacterium.

The importance of the nutritional environment for $S$. flexneri virulence is documented by the involvement in virulence of $\mathrm{fts} Z$ (Mac Síomóin et al., 1996), a gene whose expression is affected in $E$. coli by the growth medium (Powell \& Court, 1998). Supplementation of quinolinate, which is an intermediate in de novo NAD biosynthesis, during invasion, reduces 100 -fold or more the invasive ability of the four Shigella species and of enteroinvasive $E$. coli (Prunier et al., 2007). However, presence of quinolate in the growth medium reduces the level of IpaB and IpaC only twofold. Prunier et al. (2007) suggested that the drastic influence of quinolinate on invasion is not caused primarily by the expression of these invasion genes but rather by some impairment in the secretion by the T3SS.

The involvement of specific nutrients in virulence systems is not unique to S. flexneri (Beatty et al., 1994; Domergue et al., 2005; Fox \& Bzik, 2002; Pfefferkorn, 1984). It is striking that to observe an influence by the nutritional environment requires judicious and specific supplementa- 
tions. Nutritional control may prevent wasteful production of secreted virulence factors and ensure a strong and appropriate activation when this is required, as for other environmental controls (Porter \& Dorman, 1997). The composition of the luminal content changing along the digestive tract may favour the pathogenic behaviour of a contaminating Shigella. The bacterium may first encounter the deprivation of the inhibitory ornithine, which may be absorbed by intestinal receptors before it meets the increased availability of polyamines, supporting the bacterial population occurring in the colon. Reciprocally, intentional governing of nutritional content by providing specific metabolites or small-molecule effectors may confound or redirect the bacterial expression of key virulence factors to prevent the onset of the disease. Ornithine, pharmacologically supplied in the colon, might, for example, beneficially interfere in vivo with the production of pathogenic factors. The unexpected sensitivity of virF expression to Orn identifies the most upstream regulator of virulence as a target to changes in specific part(s) of the metabolism. This sensitivity may be a feature of the original host from which virF has probably been transferred horizontally. In this regard, the similarity of virF to the oruR gene of Pseudomonas aeruginosa, another transcriptional activator of the AraC family, involved in ornithine catabolism (Hebert \& Houghton, 1997), should be noted as offering another potential area of study for corresponding nutrient effects. Schuhmacher \& Klose (1999) have shown that for $V$. cholerae, the ToxTdependent transcriptional activity of virulence-associated genes is modulated by environmental signals such as temperature and presence of bile salts. The hypothetical influence of these environmental features on translation of the toxT mRNA was not evoked as a possible explanation for these modulations in $V$. cholerae. However, the suggested interference by environmental molecules in the activity of the metabolism controlled by the ToxT protein, which, like VirF of Shigella, belongs to the AraC transcriptional regulators, may very well apply to the VirF regulated metabolism in Shigella.

Knowledge of VirF in S. flexneri as a common target for nutritional intake and for environmental influences indicates that the environment can affect virulence gene expression by regulating metabolic pathways identified in this study. It is not surprising therefore that in vitro factors known to influence nutritional intake and the overall metabolic status - $\mathrm{pH}$, temperature, osmolarity, and mutations in critical pathways like tRNA modifications - have the potential to interfere with virulence gene expression.

'Success' for a bacterium in general means the ability to multiply, whereas for a pathogen it is also the ability to multiply in the suitable host with proper timing. Seeing the host as a medium has been exploited in strategies for the in vivo selection of virulence genes, such as IVET (Mahan et al., 1995). Here we have shown that the composition of the growth environment is a critical factor for the success of $S$. flexneri as a pathogen. The adaptation of the bacterial metabolism represents information on the surroundings of the pathogen. Our data show that the invasive system of $S$. flexneri, in particular, captures and exploits this metabolic information by either delivering or withholding the virulence response, even as other environmental requirements such as temperature, osmolarity and $\mathrm{pH}$, are met. At the least, this may be critical for the expression of the immunogenic components expected from living vaccine strains as well as for the efficiency of Shigella-based DNA vaccines (Xu \& Ulmer, 2003). Most importantly, a large part of our knowledge on the physiopathology of S. flexneri and related organisms appears to depend on features conditioned by the quality of the nutritional surroundings. Thus, this influence, suspected since the origins of bacteriology (Pasteur, 1880), must be taken into account to obtain control over diseases.

\section{ACKNOWLEDGEMENTS}

This work was supported by grants from the Swedish Science Research council (Project BU-2930) and the Carl Trygger Foundation. T. Pál is acknowledged for the gift of antiserum and H. Watanabe and A. T. Maurelli are acknowledged for the gifts of strains and plasmids. We also thank Maria Fällman, Umeå, for her help with the invasion assay. We thank Joakim Näsvall, Umeå, Bradford Powell, Frederick (MD, USA), Bernt Eric Uhlin, Umeå, and Hans Wolf-Watz, Umeå, for discussions and critical reading of the manuscript.

\section{REFERENCES}

Beatty, W. L., Belanger, T. A., Desai, A. A., Morrison, R. P. \& Byrne, G. I. (1994). Tryptophan depletion as a mechanism of gamma interferon-mediated chlamydial persistence. Infect Immun 62, 37053711.

Berger, E. A. \& Heppel, L. A. (1972). A binding protein involved in the transport of cystine and diaminopimelic acid in Escherichia coli. J Biol Chem 247, 7684-7694.

Byrne, G. I., Lehmann, L. K. \& Landry, G. J. (1986). Induction of tryptophan catabolism is the mechanism for gamma-interferonmediated inhibition of intracellular Chlamydia psittaci replication in T24 cells. Infect Immun 53, 347-351.

Casalino, M., Latella, M. C., Prosseda, G. \& Colonna, B. (2003). CadC is the preferential target of a convergent evolution driving enteroinvasive Escherichia coli toward a lysine decarboxylase-defective phenotype. Infect Immun 71, 5472-5479.

Celis, T. F., Rosenfeld, H. J. \& Maas, W. K. (1973). Mutant of Escherichia coli K-12 defective in the transport of basic amino acids. J Bacteriol 116, 619-626.

Cersini, A., Salvia, A. M. \& Bernardini, M. L. (1998). Intracellular multiplication and virulence of Shigella flexneri auxotrophic mutants. Infect Immun 66, 549-557.

Domergue, R., Castaño, I., De Las Peñas, A., Zupancic, M., Lockatell, V., Hebel, J. R., Johnson, D. \& Cormack, B. P. (2005). Nicotinic acid limitation regulates silencing of Candida adhesins during UTI. Science 308, 866-870.

Dorman, C. J. \& Porter, M. E. (1998). The Shigella virulence gene regulatory cascade: a paradigm of bacterial gene control mechanisms. Mol Microbiol 29, 677-684. 
Durand, J. M. \& Björk, G. R. (2003). Putrescine or a combination of methionine and arginine restores virulence gene expression in a tRNA modification-deficient mutant of Shigella flexneri: a possible role in adaptation of virulence. Mol Microbiol 47, 519-527.

Durand, J. M., Okada, N., Tobe, T., Watarai, M., Fukuda, I., Suzuki, T., Nakata, N., Komatsu, K., Yoshikawa, M. \& Sasakawa, C. (1994). $v a c C$, a virulence-associated chromosomal locus of Shigella flexneri, is homologous to tgt, a gene encoding tRNA-guanine transglycosylase (Tgt) of Escherichia coli K-12. J Bacteriol 176, 4627-4634.

Durand, J. M. B., Björk, G. R., Kuwae, A., Yoshikawa, M. \& Sasakawa, C. (1997). The modified nucleoside 2-methylthio- N-6-isopentenyladenosine in tRNA of Shigella flexneri is required for expression of virulence genes. J Bacteriol 179, 5777-5782.

Durand, J. M., Dagberg, B., Uhlin, B. E. \& Björk, G. R. (2000). Transfer RNA modification, temperature and DNA superhelicity have a common target in the regulatory network of the virulence of Shigella flexneri: the expression of the virF gene. Mol Microbiol 35, 924-935.

Emilsson, V. \& Kurland, C. G. (1990). Growth rate dependence of transfer RNA abundance in Escherichia coli. EMBO J 9, 4359-4366.

Formal, S. B., Gemski, P., Baron, L. S. \& Labrec, E. H. (1971). A chromosomal locus which controls the ability of Shigella flexneri to evoke keratoconjunctivitis. Infect Immun 3, 73-79.

Fox, B. A. \& Bzik, D. J. (2002). De novo pyrimidine biosynthesis is required for virulence of Toxoplasma gondii. Nature 415, 926-929.

Gehrke, C. W. \& Kuo, K. C. (1989). Ribonucleoside analysis by reversed-phase high-performance liquid chromatography. J Chromatogr 471, 3-36.

Gehrke, C. W., Kuo, K. C., McCune, R. A., Gerhardt, K. O. \& Agris, P. F. (1982). Quantitative enzymatic hydrolysis of tRNAs: reversedphase high-performance liquid chromatography of tRNA nucleosides. J Chromatogr 230, 297-308.

Glansdorff, N. (1996). Biosynthesis of arginine and polyamines. In Escherichia coli and Salmonella: Cellular and Molecular Biology, 2nd edn, pp. 408-433. Edited by F. C. Neidhardt and others. Washington, DC: American Society for Microbiology.

Hale, T. L. \& Formal, S. B. (1981). Protein synthesis in HeLa or Henle 407 cells infected with Shigella dysenteriae 1, Shigella flexneri 2a, or Salmonella typhimurium W118. Infect Immun 32, 137-144.

Hebert, M. D. \& Houghton, J. E. (1997). Regulation of ornithine utilization in Pseudomonas aeruginosa (PAO1) is mediated by a transcriptional regulator, OruR. J Bacteriol 179, 7834-7842.

Karlsson, S., Burman, L. G. \& Akerlund, T. (1999). Suppression of toxin production in Clostridium difficile VPI 10463 by amino acids. Microbiology 145, 1683-1693.

Mac Síomóin, R. A., Nakata, N., Murai, T., Yoshikawa, M., Tsuji, H. \& Sasakawa, C. (1996). Identification and characterization of ispA, a Shigella flexneri chromosomal gene essential for normal in vivo cell division and intracellular spreading. Mol Microbiol 19, 599-609.

Mahan, M. J., Tobias, J. W., Slauch, J. M., Hanna, P. C., Collier, R. J. \& Mekalanos, J. J. (1995). Antibiotic-based selection for bacterial genes that are specifically induced during infection of a host. Proc Natl Acad Sci U S A 92, 669-673.

Maurelli, A. T. \& Sansonetti, P. J. (1988). Identification of a chromosomal gene controlling temperature-regulated expression of Shigella virulence. Proc Natl Acad Sci U S A 85, 2820-2824.

Maurelli, A. T., Blackmon, B. \& Curtiss, R. (1984). Temperaturedependent expression of virulence genes in Shigella species. Infect Immun 43, 195-201.

Maurelli, A. T., Fernández, R. E., Bloch, C. A., Rode, C. K. \& Fasano, A. (1998). "Black holes" and bacterial pathogenicity: a large genomic deletion that enhances the virulence of Shigella spp. and enteroinvasive Escherichia coli. Proc Natl Acad Sci U S A 95, 3943-3948.

Miller, J. H. (1972). Experiments in Molecular Genetics. Cold Spring Harbor, NY: Cold Spring Harbor Laboratory.

Miller, V. L. \& Mekalanos, J. J. (1988). A novel suicide vector and its use in construction of insertion mutations: osmoregulation of outer membrane proteins and virulence determinants in Vibrio cholerae requires toxR. J Bacteriol 170, 2575-2583.

Miller, J. F., Mekalanos, J. J. \& Falkow, S. (1989). Coordinate regulation and sensory transduction in the control of bacterial virulence. Science 243, 916-922.

Nakata, N., Sasakawa, C., Okada, N., Tobe, T., Fukuda, I., Suzuki, T., Komatsu, K. \& Yoshikawa, M. (1992). Identification and characterization of virK, a virulence-associated large plasmid gene essential for intercellular spreading of Shigella flexneri. Mol Microbiol 6, 23872395.

Nakayama, S. \& Watanabe, H. (1995). Involvement of $c p x A$, a sensor of a two-component regulatory system, in the pH-dependent regulation of expression of Shigella sonnei virF gene. J Bacteriol 177, 5062-5069.

Neidhardt, F. C., Bloch, P. L. \& Smith, D. F. (1974). Culture medium for enterobacteria. J Bacteriol 119, 736-747.

Neidhardt, F. C., Bloch, P. L., Pedersen, S. \& Reeh, S. (1977). Chemical measurement of steady-state levels of ten aminoacyltransfer ribonucleic acid synthetases in Escherichia coli. J Bacteriol 129, 378-387.

Neidhardt, F. C., Curtiss, R., III, Ingraham, J. L., Lin, E. C. C., Low, K. B., Jr, Magasanik, B., Reznikoff, W. S., Riley, M., Schaechter, M. \& Umbarger, H. E. (1996). Escherichia coli and Salmonella: Cellular and Molecular Biology, 2nd edn. Washington, DC: American Society for Microbiology.

Noriega, F. R., Losonsky, G., Lauderbaugh, C., Liao, F. M., Wang, J. Y. \& Levine, M. M. (1996). Engineered $\Delta g u a B-A \Delta v i r G$ Shigella flexneri $2 \mathrm{a}$ strain CVD 1205: construction, safety, immunogenicity, and potential efficacy as a mucosal vaccine. Infect Immun 64, 3055-3061.

Ogawa, M. \& Sasakawa, C. (2006). Intracellular survival of Shigella. Cell Microbiol 8, 177-184.

Okada, N., Sasakawa, C., Tobe, T., Yamada, M., Nagai, S., Talukder, K. A., Komatsu, K., Kanegasaki, S. \& Yoshikawa, M. (1991). Virulence-associated chromosomal loci of Shigella flexneri identified by random Tn5 insertion mutagenesis. Mol Microbiol 5, 187-195.

Pál, T., Formal, S. B. \& Hale, T. L. (1989). Characterization of virulence marker antigen of Shigella spp. and enteroinvasive Escherichia coli. JClin Microbiol 27, 561-563.

Pasteur, L. (1880). De l'attenuation du virus du choléra des poules. C R Acad Sci 91, 673-680.

Pfefferkorn, E. R. (1984). Interferon gamma blocks the growth of Toxoplasma gondii in human fibroblasts by inducing the host cells to degrade tryptophan. Proc Natl Acad Sci U S A 81, 908-912.

Porter, M. E. \& Dorman, C. J. (1994). A role for H-NS in the thermoosmotic regulation of virulence gene expression in Shigella flexneri. J Bacteriol 176, 4187-4191.

Porter, M. E. \& Dorman, C. J. (1997). Differential regulation of the plasmid-encoded genes in the Shigella flexneri virulence regulon. Mol Gen Genet 256, 93-103.

Powell, B. S. \& Court, D. L. (1998). Control of $f t s Z$ expression, cell division, and glutamine metabolism in Luria-Bertani medium by the alarmone ppGpp in Escherichia coli. J Bacteriol 180, 1053-1062.

Prunier, A. L., Schuch, R., Fernández, R. E., Mumy, K. L., Kohler, H., McCormick, B. A. \& Maurelli, A. T. (2007). nadA and nadB of Shigella flexneri $5 \mathrm{a}$ are antivirulence loci responsible for the synthesis of 
quinolinate, a small molecule inhibitor of Shigella pathogenicity. Microbiology 153, 2363-2372.

Rietsch, A. \& Mekalanos, J. J. (2006). Metabolic regulation of type III secretion gene expression in Pseudomonas aeruginosa. Mol Microbiol 59, 807-820.

Rothman, S. W. \& Corwin, L. M. (1972). Factors affecting virulence of Shigella flexneri: defective methionine synthesis in an Escherichia coliShigella hybrid. J Bacteriol 112, 176-182.

Sansonetti, P. J., Ryter, A., Clerc, P., Maurelli, A. T. \& Mounier, J. (1986). Multiplication of Shigella flexneri within HeLa cells: lysis of the phagocytic vacuole and plasmid-mediated contact hemolysis. Infect Immun 51, 461-469.

Sasakawa, C., Kamata, K., Sakai, T., Murayama, S. Y., Makino, S. \& Yoshikawa, M. (1986). Molecular alteration of the 140-megadalton plasmid associated with loss of virulence and Congo red binding activity in Shigella flexneri. Infect Immun 51, 470-475.
Schuhmacher, D. A. \& Klose, K. E. (1999). Environmental signals modulate ToxT-dependent virulence factor expression in Vibrio cholerae. J Bacteriol 181, 1508-1514.

Tobe, T., Nagai, S., Okada, N., Adler, B., Yoshikawa, M. \& Sasakawa, C. (1991). Temperature-regulated expression of invasion genes in Shigella flexneri is controlled through the transcriptional activation of the $\operatorname{vir} B$ gene on the large plasmid. Mol Microbiol 5, 887-893.

Xu, F. \& Ulmer, J. B. (2003). Attenuated Salmonella and Shigella as carriers for DNA vaccines. J Drug Target 11, 481-488.

Zagaglia, C., Casalino, M., Colonna, B., Conti, C., Calconi, A. \& Nicoletti, M. (1991). Virulence plasmids of enteroinvasive Escherichia coli and Shigella flexneri integrate into a specific site on the host chromosome: integration greatly reduces expression of plasmidcarried virulence genes. Infect Immun 59, 792-799.

Edited by: P. H. Everest 